\title{
PADRÕES ISOENZIMÁTICOS E MORFOLOGIA DE ISOLADOS DE Alternaria spp. PATOGÊNICOS A Senna obtusifolia
}

\author{
SUELI C. M. MELLO ${ }^{1}$, ZILDA M. A. RIBEIRO ${ }^{1}$, GUTEMBERG R. SOUSA ${ }^{1}$, MYRIAN TIGANO ${ }^{1}$, GLAUCIA DE \\ F. NACHTIGAL ${ }^{2} \&$ ELIANA MARIA G. FONTES ${ }^{1}$
}

\author{
${ }^{1}$ Embrapa/ Recursos Genéticos e Biotecnologia, Cx. Postal 02372, CEP 70770-900, Brasília, DF, Fax (061) 348-4776, \\ e-mail: smello@cenargen.embrapa.br; ${ }^{2}$ Universidade Camilo Castelo Branco, Faculdade de Ciências Agrárias, \\ Campus VII, CEP 15600-000, Fernandópolis, SP
}

(Aceito para publicação em 31/05/2001)

Autor para correspondência: Sueli C. M. de Mello

MELlO, S.C.M., RIBEIRO, Z.M.A., SOUSA, G.R., TIGANO, M., CACHTIGAL, F.G. \& FONTES, E.M.G. Padrões isoenzimáticos e morfologia de isolados de Alternaria spp. patogênicos a Senna obtusifolia. Fitopatologia Brasileira 26:667-669. 2001.

\section{RESUMO}

Isolados de Alternaria spp. patogênicos ao fedegoso (Senna obtusifolia) foram estudados quanto aos aspectos morfológicos e padrões isoenzimáticos, em gel de poliacrilamida. Com base nas características culturais, morfologia de colônias e morfometria de conídios, verificou-se que 11 dos 13 isolados pertenciam à espécie $A$. cassiae e os outros dois à A. alternata. Os resultados da análise eletroforética corroboraram as informações obtidas por meio de critérios morfológicos. Portanto, esta técnica tem potencial para ser usada na separação destas espécies de Alternaria. A ocorrência de mais de uma espécie deste gênero fúngico em $S$. obtusifolia foi constatada, ampliando o número de patógenos a serem avaliados no programa de desenvolvimento de um bioherbicida para esta espécie de planta daninha.

Palavras-chave: biocontrole, patógeno, planta daninha, caracterização.

\section{ABSTRACT \\ Isoenzyme patterns and morphology of isolates of Alternaria species pathogenic to Senna obtusifolia}

Alternaria spp. isolates pathogenic to sicklepod (Senna obtusifolia) were characterized based on morphological characteristics and isozyme patterns. Colonial morphology and conidial shape showed that 11 of the 13 isolates belong to A. cassiae. The other two isolates were identified as $A$. alternata. Isoenzyme patterns confirmed the results of morphological criteria. The occurrence of more than one species of this fungus infecting S. obtusifolia was found, widening the range of suitable pathogens to be evaluated in bioherbicide programs for sicklepod control.
Um grande número de espécies de Alternaria tem sido relatado causando manchas foliares em diversas plantas cultivadas e não cultivadas, dentre estas, algumas espécies daninhas que infestam culturas economicamente importantes. Um bioherbicida foi desenvolvido a partir de Alternaria cassiae Jurair \& Khan (Simons, 1982) e registrado nos Estados Unidos para controle do fedegoso (Senna obtusifoliaI L.) (Charudattan, 1996). No Brasil, onde esta planta daninha vem-se tornando um sério problema, particularmente em áreas cultivadas com soja [Glycine max (L.) Merril], um isolado de A. cassiae, coletado no Distrito Federal (DF), demonstrou grande potencial para ser utilizado contra biotipos brasileiros desta planta (Figueiredo et al., 1992). Outros isolados de Alternaria spp. foram obtidos de folhas e hastes de $S$. obtusifolia, coletadas em áreas do DF, em diferentes ocasiões, e incorporados à Coleção de Fungos Agentes de Controle Biológico da Embrapa-Recursos Genéticos e Biotecnologia, Brasília, DF. Este trabalho teve como objetivos caracterizar esses isolados por meio de características morfológicas e análise de isoenzimas em gel de poliacrilamida e do estudo das características morfológicas.

Foram utilizadas culturas monospóricas de 13 isolados de Alternaria spp. (Tabela 1), dentre os quais, o isolado CG594, anteriormente identificado como A. cassiae (Figueiredo et al., 1992). Estes isolados foram previamente avaliados quanto à patogenicidade, nas hospedeiras originais e mantidos em tubos de ensaio contendo o meio de batatadextrose-agar (BDA) a $4{ }^{\circ} \mathrm{C}$. Um isolado de Alternaria solani Sorauer (As), obtido de tomate (Lycopersicon esculentum Mill.), cedido pela Embrapa-Hortaliças, Brasília, DF, foi incluído como referência desta espécie.

Para os estudos morfológicos, colônias foram desenvolvidas em placas de Petri contendo BDA, a $28{ }^{\circ} \mathrm{C}$, sob fotoperíodo de $12 \mathrm{~h}$. O fotoperíodo foi simulado por meio de quatro lâmpadas fluorescentes de $20 \mathrm{~W}$, luz do dia, instaladas na porta da BOD (Nova Técnica, modelo NT 708 - AT). Observações quanto ao aspecto de micélio aéreo e 
S.C.M. Mello et al.

TABELA 1 - Morfometria de conídios de isolados de Alternaria spp.

\begin{tabular}{lccccc}
\hline Isolado & $\begin{array}{c}\text { Comp. x Larg. } \\
\text { Conídio }(\boldsymbol{\mu m}) \mathbf{1}^{\mathbf{1}}\end{array}$ & $\begin{array}{c}\text { Comp. Med. } \\
\text { Conídio }(\boldsymbol{\mu m})^{2}\end{array}$ & $\begin{array}{c}\text { Conídio + bico } \\
(\boldsymbol{\mu m})^{\mathbf{2}}\end{array}$ & $\begin{array}{c}\text { Larg. Med. } \\
\text { Conídio }(\boldsymbol{\mu m})^{3}\end{array}$ & $\begin{array}{c}\text { Septo transversal } \\
(\boldsymbol{\mu m})^{\mathbf{4}}\end{array}$ \\
\hline CG594 & $90,2-241,4 \times 12,2-26,8$ & $65,5 \mathrm{~b}$ & $141,3 \mathrm{~cd}$ & $18,7 \mathrm{bc}$ & $6,872 \mathrm{bcd}$ \\
CG598 & $26,8-09,7 \times 9,7-17$ & $27,4 \mathrm{e}$ & $55,7 \mathrm{~g}$ & $12,8 \mathrm{~g}$ & $3,925 \mathrm{~g}$ \\
CG600 & $97,5-180,5 \times 9,7-21,9$ & $58,4 \mathrm{c}$ & $144,4 \mathrm{~cd}$ & $15,0 \mathrm{f}$ & $6,825 \mathrm{~cd}$ \\
CG601 & $148,7-19,5 \times 17,0-21,9$ & $70,1 \mathrm{a}$ & $178,7 \mathrm{a}$ & $20,3 \mathrm{a}$ & $7,525 \mathrm{a}$ \\
CG603 & $100-226,9 \times 12,2-29,3$ & $64,4 \mathrm{~b}$ & $153,2 \mathrm{bc}$ & $19,3 \mathrm{ab}$ & $7,625 \mathrm{a}$ \\
CG604 & $36,6-92,7 \times 7,3-14,6$ & $28,3 \mathrm{e}$ & $57,1 \mathrm{~g}$ & $11,4 \mathrm{~h}$ & $4,775 \mathrm{f}$ \\
CG606 & $65,9-231,7 \times 9,7-19,5$ & $55,4 \mathrm{c}$ & $63,0 \mathrm{~b}$ & $14,8 \mathrm{f}$ & $7,125 \mathrm{abc}$ \\
CG607 & $92,6-190,2 \times 12,2-26,8$ & $66,4 \mathrm{ab}$ & $139,3 \mathrm{~d}$ & $16,5 \mathrm{de}$ & $7,400 \mathrm{ab}$ \\
CG609 & $92,7-139 \times 14,6-24,4$ & $58,8 \mathrm{c}$ & $112,4 \mathrm{f}$ & $19,5 \mathrm{ab}$ & $6,175 \mathrm{e}$ \\
CG610 & $107,3-46,3 \times 12,2-24,4$ & $49,5 \mathrm{~d}$ & $136,2 \mathrm{~d}$ & $17,9 \mathrm{c}$ & $6,150 \mathrm{e}$ \\
CG611 & $107,3-07,3 \times 9,7-21,9$ & $54,3 \mathrm{c}$ & $164,4 \mathrm{~b}$ & $16,0 \mathrm{ef}$ & $6,475 \mathrm{de}$ \\
CG615 & $75,6-180,5 \times 12,2-19,5$ & $54,7 \mathrm{c}$ & $120,2 \mathrm{ef}$ & $15,0 \mathrm{f}$ & $7,675 \mathrm{a}$ \\
CG616 & $100-160,9 \times 14,6-24,4$ & $57,8 \mathrm{c}$ & $131,9 \mathrm{de}$ & $17,5 \mathrm{~cd}$ & $6,878 \mathrm{bcd}$ \\
\hline C.V. $(\%)$ & & 0,1671 & 0,2169 & 0,1691 & 0,1764 \\
\hline
\end{tabular}

${ }^{1}$ Valores mínimo e máximo encontrados para comprimento e largura de 40 conídios, tomados ao acaso

${ }^{2}$ Comprimento médio de 40 conídios, tomados ao acaso

${ }^{3}$ Largura média de 40 estruturas, tomadas ao acaso

${ }^{4}$ Número médio de septos de 40 conídios, tomados ao acaso

submerso, bem como de presença de esporulação, foram feitas durante o período de cultivo. Após dez dias de crescimento, as colônias tiveram seu micélio aéreo removido e foram expostas ao ar, para esporulação.

As estruturas dos fungos foram montadas em lâminas com glicerol $+\mathrm{KOH}$ e observadas ao microscópio de luz. De cada isolado, foram utilizados 40 esporos para contagem de septos e medições do comprimento do conídio, comprimento total (conídio + bico) e largura do conídio. Os dados obtidos foram submetidos à análise de variância e as médias, comparadas pelo teste de Duncan, por meio do pacote estatístico MSTATC (Russel P. Feed, Michigan State University).

Para análise de isoenzimas, o micélio para extração de proteínas foi produzido em meio semi-sintético preparado a partir de $10 \mathrm{~g}$ de sacarose, $2 \mathrm{~g}$ de L-asparagina, $2 \mathrm{~g}$ de extrato de levedura, $1 \mathrm{~g}$ de $\mathrm{KH}_{2} \mathrm{PO}_{4}, 0,1 \mathrm{~g}$ de $\mathrm{MgSO}_{4}-7 \mathrm{H}_{2} \mathrm{O}$, $0,44 \mathrm{mg} \mathrm{ZnSO}_{4}-7 \mathrm{H}_{2} \mathrm{O}, 0,48 \mathrm{mg} \mathrm{FeCl}-6 \mathrm{H}_{2} \mathrm{O}, 0,36 \mathrm{mg}$ $\mathrm{MnCl}^{2}-\mathrm{H}_{2} \mathrm{O}$ em 11 de água destilada, $\mathrm{pH} 5,3$ (Alfenas et al., 1991). Frascos Erlenmeyers, contendo $50 \mathrm{ml}$ do meio receberam cinco discos de micélio retirados de colônias com sete dias de idade. Os frascos foram mantidos em estufa incubadora (Nova Técnica, modelo NT 708 - AT) a $28{ }^{\circ} \mathrm{C}$, no escuro, durante 14 dias. Após este período, o micélio foi coletado por filtração através de papel de filtro (Whatman fi $\mathrm{N}^{\circ} 1$ ), lavado várias vezes em água destilada esterilizada e, quando não utilizado imediatamente, estocado a $-80{ }^{\circ} \mathrm{C}$.

Para extração das proteínas e realização da eletroforese, seguiram-se os procedimentos descritos por Alfenas et al., (1991). Testaram-se os seguintes sistemas enzimáticos: Enzima Málica (ME) EC 1.1.1.40, Esterase (EST) EC 3.1.1.1., Sorbitol Desidrogenase (SDH) EC 1.1.1.14, Menadiona Redutase (MR) EC 1.6.99.2, Fosfatase Alcalina (ALP) EC 3.1.3.1, 6-Fosfogluconato Desidrogenase (PGDH) EC 1.1.1.44, Manitol Desidrogenase (MADH) EC
1.1.1.67, Fosfatase ácida (ACP) EC 3.1.3.2, Diaforase (DIA) EC 1.8.1.4, Nicotinamida Adenina Dinucleotídeo Desidrogenase (NADHDH) EC 1.6.99.3, Superóxido Dismutase (SOD) EC 1.15.1.1, Polifenoloxidase (PPO) EC 1.14.18.1, Álcool Desidrogenase (ADH) EC 1.1.1.1, $\beta$ Galactose Desidrogenase (GLDH) EC 1.1.1.48 e Malato Desidrogenase (MDH) EC 1.1.1.37. Após a eletroforese, os géis foram corados para deteç̧ão de atividade isoenzimática, conforme Alfenas et al., (1991).

A partir dos fenótipos isoenzimáticos encontrados, os isolados foram agrupados pelo método não ponderado de agrupamento aos pares ("Unweighted Pair Group Method With Arithmetic Averages-U.P.G.M.A), utilizando-se o pacote NTSYS-pc para taxonomia numérica e análise multivariada (Rohlf, 1993). A matriz de similaridade foi calculada pelo coeficiente de Jaccard.

Todos os isolados cresceram prontamente em BDA, sendo as colônias características do gênero Alternaria, de acordo com a descrição feita por Ellis (1971). As colônias dos isolados CG598 e CG604 cresceram rapidamente, tomando toda a superfície da placa, de forma irregular e esporularam profusamente, durante o período de incubação, sem exigir nenhum tratamento estressante. Entretanto, os outros isolados formaram colônias circulares, não esporulantes e somente produziram esporos após escarificação do micélio superficial e exposição ao ar.

A análise estatística das características dos conídios estudadas revelou diferença significativa $(\mathrm{p}=0,05)$ entre os isolados (Tabela 1). Os isolados CG598 e CG604 apresentaram os menores valores médios de comprimento de conídios, comprimento de conídios + bico e largura de conídios, para os quais não se verificaram diferenças significativas entre si, tendo entretanto, diferido dos demais isolados. No tocante ao número de septos tranversais dos conídios, embora tenha sido detectada diferença significativa entre estes dois isolados, ambos apresentaram 
os menores valores médios. Nos testes de patogenicidade previamente realizados, estes dois isolados também se mostraram menos agressivos a plântulas de fedegoso (dados não publicados). Embora o comprimento dos conídios tenha diferido da descrição de Ellis (1971) no seu limite superior, as demais características observadas levaram a identificá-los como pertencentes à espécie $A$. alternata (Fr.) Keissler.

Os demais isolados, apesar de, em alguns casos, terem diferido significativamente entre si, ao nível de 0,05 de probabilidade, com relação às características estudadas (Tabela 1), mostraram-se semelhantes quanto ao crescimento, morfologia de colônias e esporulação que foram de acordo com a descrição feita por Simmons (1982) para a espécie A. cassiae. As diferenças verificadas podem ser atribuídas a vários fatores, dentre os quais, a idade do conídio (Simmons, 1982).

O fungo A. alternata tem sido relatado como saprófita e também como agente patogênico em muitas plantas (Ellis, 1971), incluíndo algumas espécies daninhas como Abutilon theophrasti Medic. (Kremer, 1986), Centaurea maculosa Lam. (Strobel, 1991), Datura stramonium L. (Abbas et al., 1993) e Epilobium angustifolium L. (Winder \& Watson, 1994), e como produtora de fitotoxinas com propriedades herbicidas (Abbas et al. 1995). A ocorrência desta espécie em S. obtusifolia, constatada neste trabalho, amplia o número de patógenos a serem avaliados no programa de bioherbicida para essa planta daninha.

No tocante aos perfis isoenzimáticos, dentre as 16 enzimas estudadas, ADH, GLDH e MDH foram as únicas que não apresentaram atividade. Entretanto, para a análise dos perfis dos isolados, foram utilizadas apenas as enzimas ACP, ALP, PPO, EST e SOD, que apresentaram melhor resolução e reprodutibilidade.

O agrupamento dos isolados, segundo os fenótipos isoenzimáticos apresentados, sugeriu a existência de três grupos distintos: grupo I, constituído apenas pelo isolado de A. solani; grupo II, abrangendo os 11 isolados identificados, com base na morfologia, como pertencentes à espécie A. cassiae; grupo III, constituído pelos isolados CG598 e CG604, classificados como pertencentes à espécie A. alternata (Figura 1). Portanto, os padrões eletroforéticos permitiram separar as espécies analisadas, corroborando as informações obtidas a partir de critérios morfológicos.

A caracterização dos isolados ao nível de espécie, baseada em critérios morfológicos, constitui-se tarefa árdua, exigindo grande disponibilidade de tempo. Entretanto, o uso de isoenzimas, que é uma técnica simples, eficiente e de baixo custo, mostrou ser útil para separação das espécies de Alternaria spp. patogênicas a S. obtusifolia, ainda que, para aplicação prática, torna-se necessária a caracterização de outras espécies com um maior número de isolados.

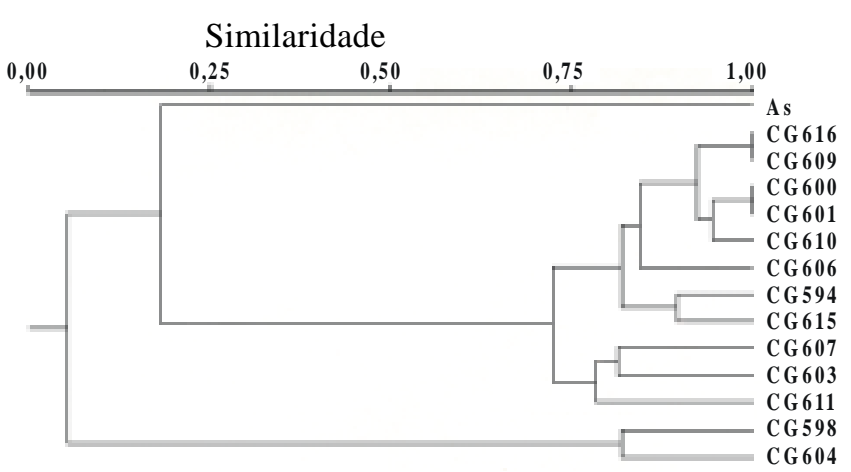

FIG. 1 - Dendrograma de isolados de Alternaria spp. patogênicos a Senna obtusifolia, obtido pelo método não ponderado de agrupamento aos pares, a partir dos fenótipos isoenzimáticos.

\section{REFERÊNCIAS BIBLIOGRÁFICAS}

ABBAS, H.K., VESONDER, R.F., BOYETTE, C.D. \& PETERSON, S.W. Phytotoxicity of AAL-toxin and other compounds produced by Alternaria alternata to jimmsonweed (Datura stramonium). Canadian Journal of Botany 71:155-160. 1993.

ABBAS, H.K., DUKE, S.O., PAUL, R.N., RYLEY, R.T. \& TANAKA, T.A. AL- toxin, a potent natural herbicide which disrupts sphingolipid metabolism of plants. Pesticide-Science 43:181-187. 1995.

ALFENAS, A.C., PETERS, I., BRUNE, W. \& PASSADOR, G.C.. Eletroforese de proteínas e isoenzimas de fungos e essências florestais. Universidade Federal de Viçosa, 1991.

CHARUDATTAN, R. Biological control of weeds: an international overview. Anais, V Simpósio de controle biológico, Foz do Iguaçu, PR. 1996. pp.270-282.

ELLIS, M.B. Dematiaceous hyphomycetes. C.A.B. Comwealth Mycological Institute Kew. Uk, 1971.

FIGUEIREDO, G.DE, FONTES, E.G., TEIXEIRA, C.A.D. \& PAIS, J.S. Levantamento e seleção de patógenos para o controle biológico de fedegoso (Senna obtusifolia : Leguminosae). Fitopatologia Brasileira 17:169, 1992. (Resumo).

KREMER, R.J. Microorganisms associated with velvetleaf (Abutilon theophrasti) seeds on soil surface. Weed Science 34:233-236. 1986.

ROHLF, F.J. NTSYS-pc: numerical taxonomy and multivariate analysis system. New York. Exeter Software, v1.1992.

SIMMONS, E.G. Alternaria themes and variations (7-10). Mycotaxon 14:17-43. 1982.

STROBEL, G.A. Biological control of weeds. Scientific American 265:50-51. 1991.

WINDER, R.S. \& WATSON, A.K. A potential microbial control for fireweed (Epilobium angustifolium). Phytoprotection 75:19-33. 1994. 